Silicon Nanoparticles with Surface Nitrogen: 90\% Quantum Yield with Narrow Luminescence Bandwidth and the Ligand Structure Based Energy Law

Qi Li, ${ }^{\dagger}$ Tian-Yi Luo, ${ }^{\ddagger}$ Meng Zhou, ${ }^{\dagger}$ Hadi Abroshan, ${ }^{\dagger}$ Jingchun Huang, ${ }^{\S}$ Hyung J. Kim, ${ }^{\dagger}, \|$ Nathaniel L. Rosi, ${ }^{\ddagger}$ Zhengzhong Shao, ${ }^{\S}$ and Rongchao Jin ${ }^{*, \dagger}$

${ }^{\dagger}$ Department of Chemistry, Carnegie Mellon University, Pittsburgh, Pennsylvania 15213, United States

${ }^{\ddagger}$ Department of Chemistry, University of Pittsburgh, Pittsburgh, Pennsylvania 15213, United States

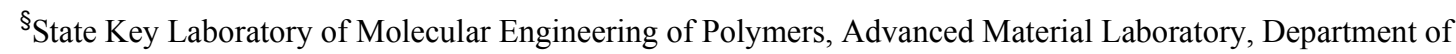
Macromolecular Science, Fudan University, Shanghai 200433, People's Republic of China

" School of Computational Sciences, Korea Institute for Advanced Study, Seoul 02455, Korea

* Email (R. Jin): rongchao@andrew.cmu.edu

\title{
List of Supporting Figures:
}

Figure S1. Size distribution of On-Si NPs.

Figure S2. PL QYs measurement of On-Si NPs.

Figure S3. PL lifetime of On Si NPs.

Figure S4. PL spectra of On Si NPs under different excitations.

Figure S5. The XPS spectrum of O 1s

Figure S6. Selected kinetic traces, the corresponding fitting and residuals obtained from the global analysis at $490 \mathrm{~nm}$.

Figure S7. Femtosecond transient absorption spectra of On-Si NPs excited at $350 \mathrm{~nm}$.

Figure S8. Selected kinetic traces, the corresponding fitting and residuals obtained from the global analysis excited at $350 \mathrm{~nm}$

Figure S9. PL and PLE spectra of Aon-Si NPs.

Figure S10. PL and PLE spectra of the Azo-Si NPs.

Figure S11. PL spectra of piperidine and 1-butylamine modified Si NPs.

Figure S12. NMR spectra of the neat On ligand and On-Si NPs.

Figure S13. NMR spectra of the neat Azo ligand and Azo-Si NPs. 
Figure S14. NMR spectra of the neat Aon ligand and Aon-Si NPs.

Figure S15. ${ }^{1} \mathrm{H}$ NMR spectra showing integration for On-Si NPs.

Figure S16. TGA plot of On-Si NPs.

Figure S17. PL, PLE and absorption spectra of the neat On ligands.

Figure S18. PL, PLE and absorption spectra of the neat Aon ligands.

Figure S19. PL, PLE and absorption spectra of the neat Azo ligands.

Figure S20. PL, PLE and absorption spectra of the neat naphthalene.

Figure S21. Photographs of the product obtained by the blank reaction using glyme, $\mathrm{Na}$ naphthalide and the capping agents alone.

Figure S22. Optimized molecular structure of (A) $\mathrm{Si}_{35} \mathrm{H}_{36}$, (B) $\mathrm{Si}_{35} \mathrm{H}_{35} \mathrm{~L}$ and (C) L, where $\mathrm{L}=$ 1, 2, 3, 4-tetrahydrocarbazol-4-one. 


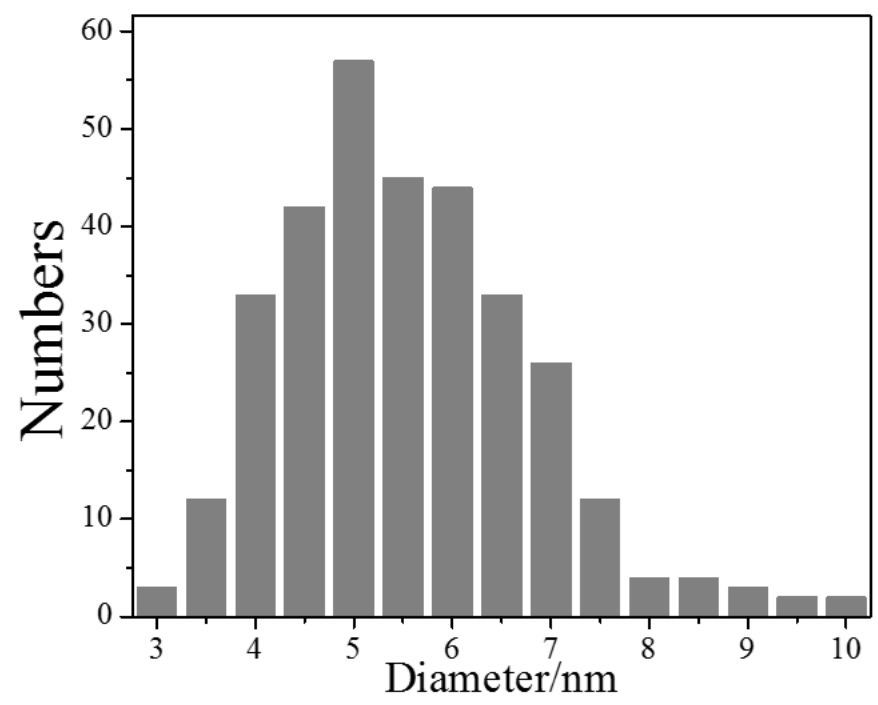

Figure S1. Size distribution of the On-Si NPs (average size: $5.2 \mathrm{~nm}$ ).

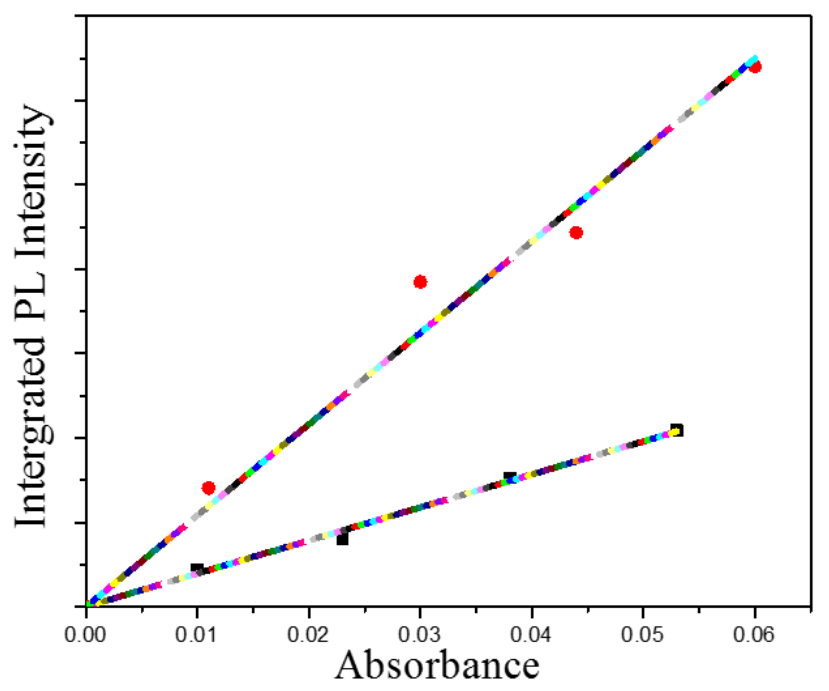

Figure S2. Measurement of On-Si NPs PL quantum yield using Rhodamine B as a reference. A typical example of integrated PL intensity dependence on the absorbance of Si NPs (red •) and Rhodamine B (black $\cdot$ ) in water. The solid lines represent the fitting results for each set of data. PLQY of Si NPs was calculated by using the equation: $\varphi_{\mathrm{Si}}=\varphi_{\text {stand. }}\left(\mathrm{k}_{\mathrm{x}} / \mathrm{k}_{\text {stand. }}\right)\left(\eta_{\mathrm{Si}} / \eta_{\text {stand. }}\right)^{2}$, where $\varphi$ is the QY, $k$ is the slope and $\eta$ is the refractive index. For aqueous solutions, $\eta_{\mathrm{Si}} / \eta_{\text {stand. }}=1$. 


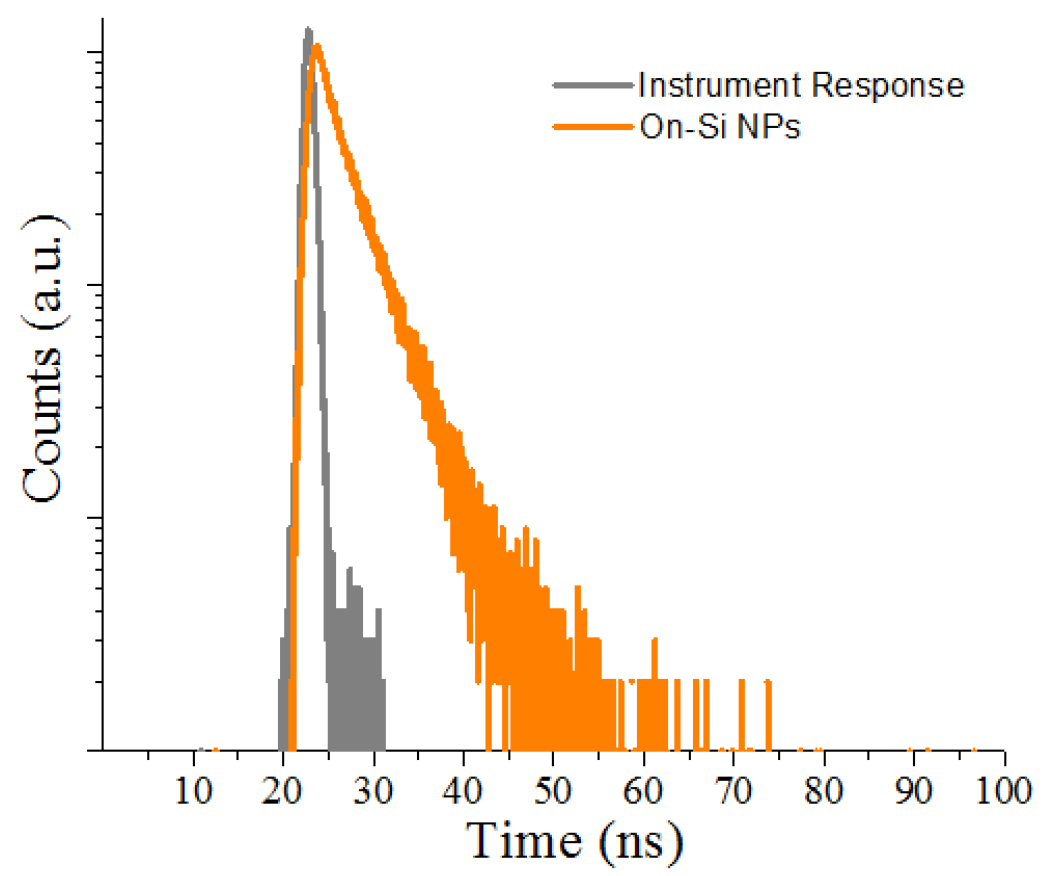

Figure S3. TCSPC measurement of On-Si NPs which shows a PL lifetime of 3 ns in methanol.

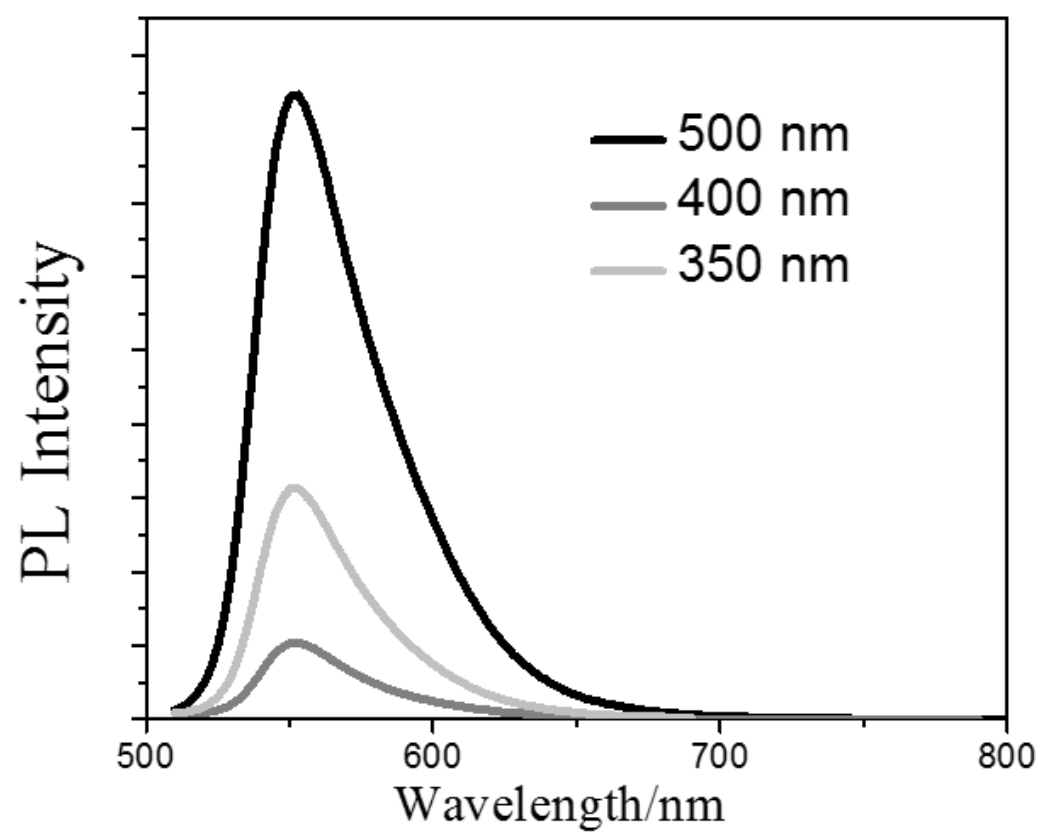

Figure S4. PL spectra of On-Si NPs under different excitation wavelengths. 


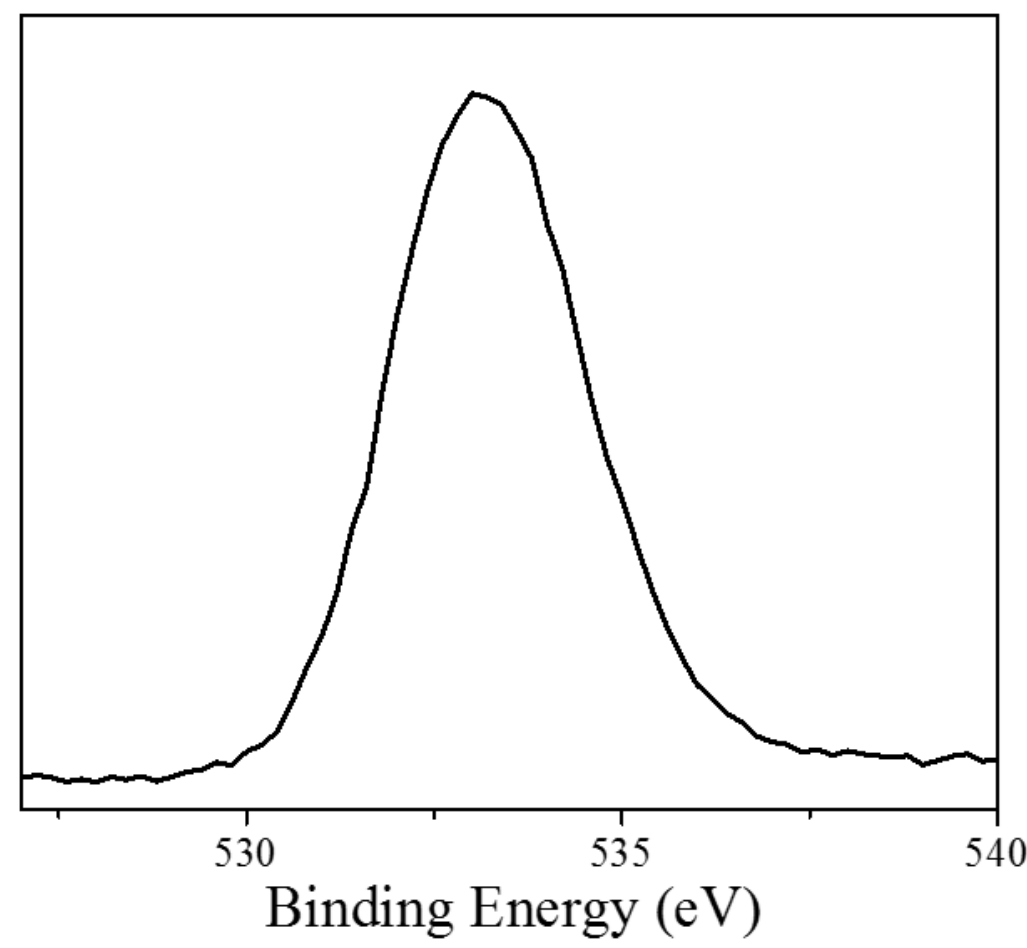

Figure S5. The O 1s region X-ray photoemission spectrum of On-Si NPs.

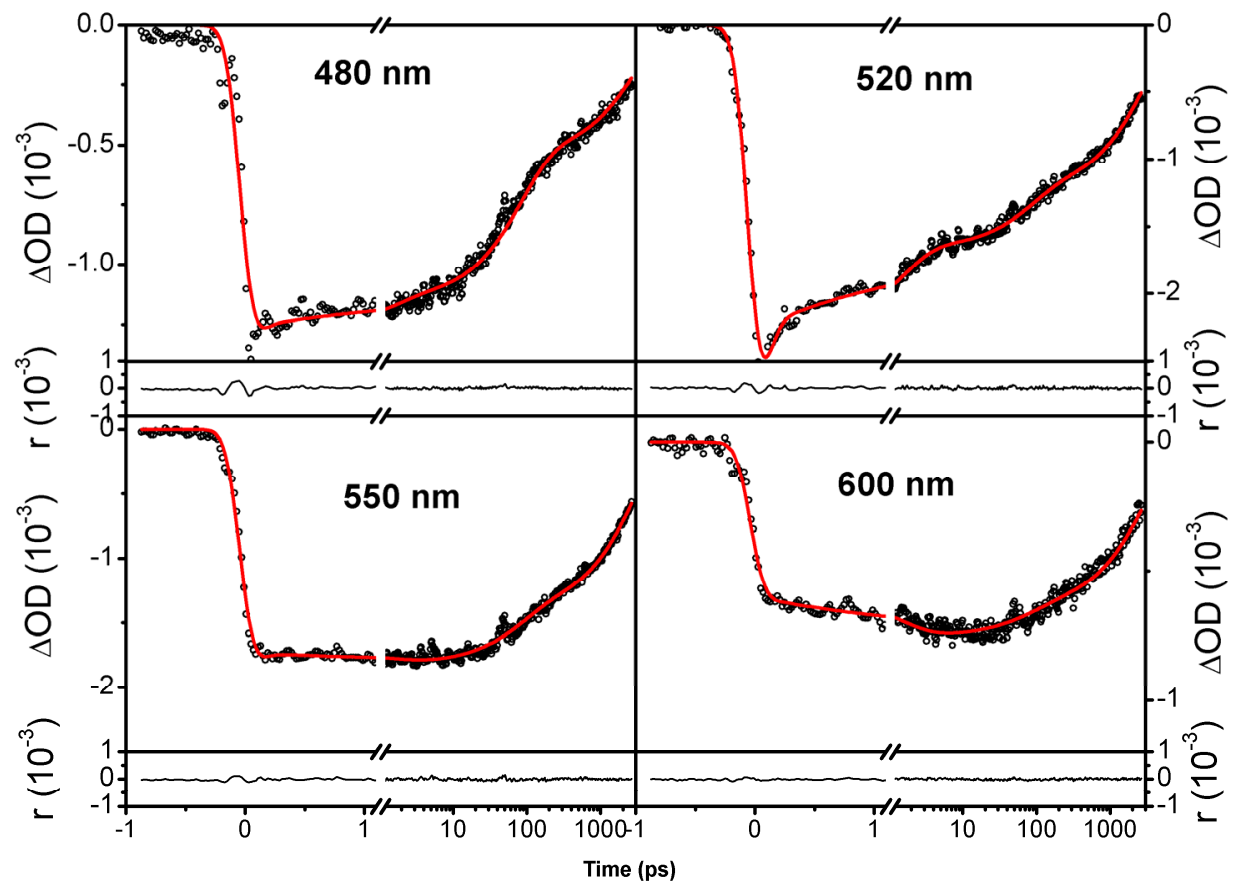

Figure S6. Selected kinetic traces, the corresponding fitting and residuals obtained from the global analysis excited at $490 \mathrm{~nm}$. 


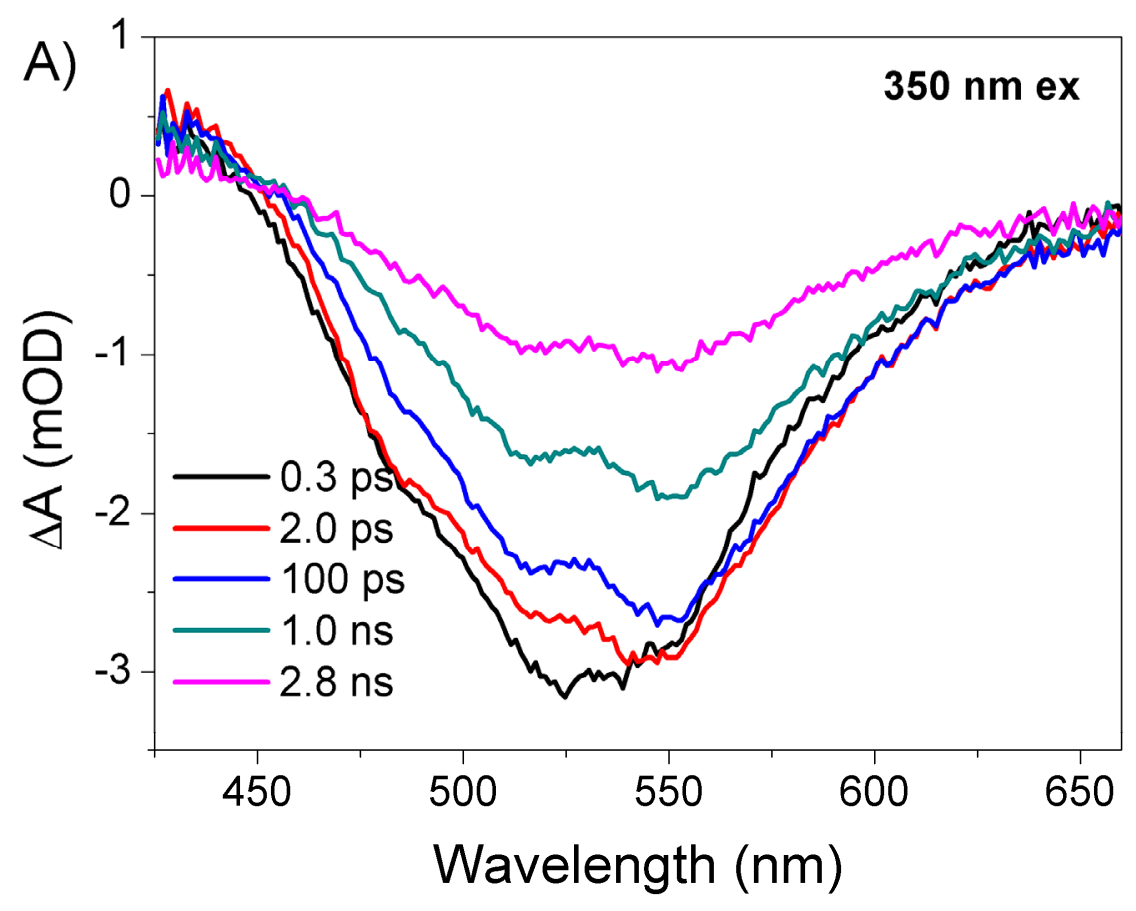

Figure S7. Time evolution of transient absorption spectra of Si NC excited at $350 \mathrm{~nm}$.

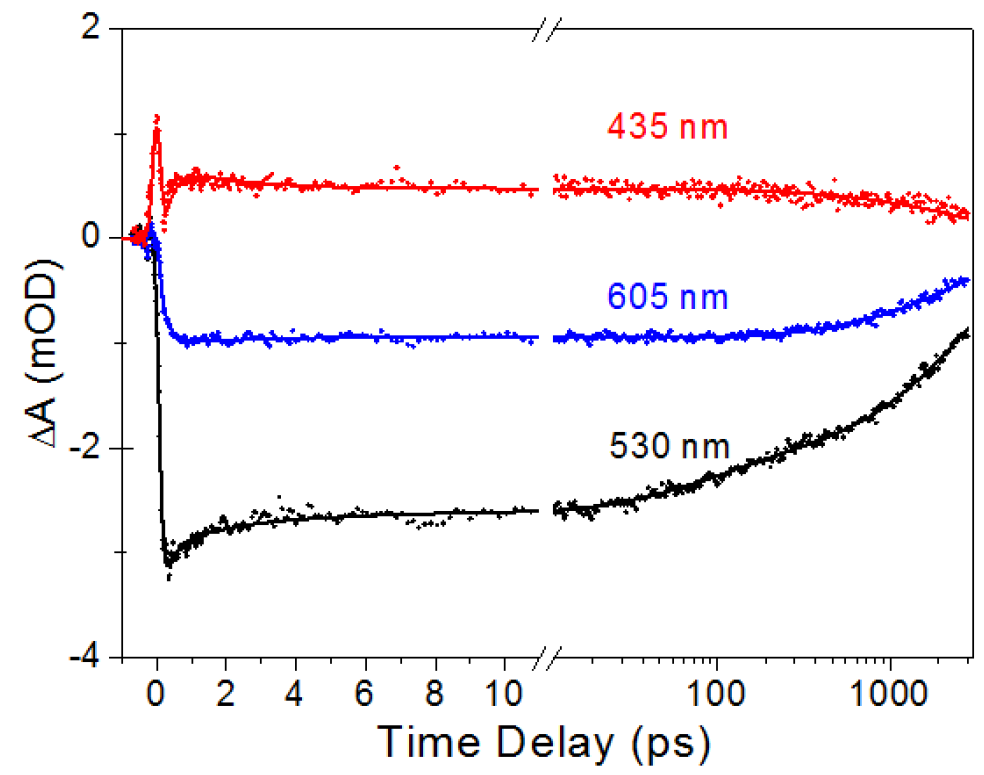

Figure S8. Selected kinetic traces, the corresponding fitting and residuals obtained from the global analysis excited at $350 \mathrm{~nm}$. 

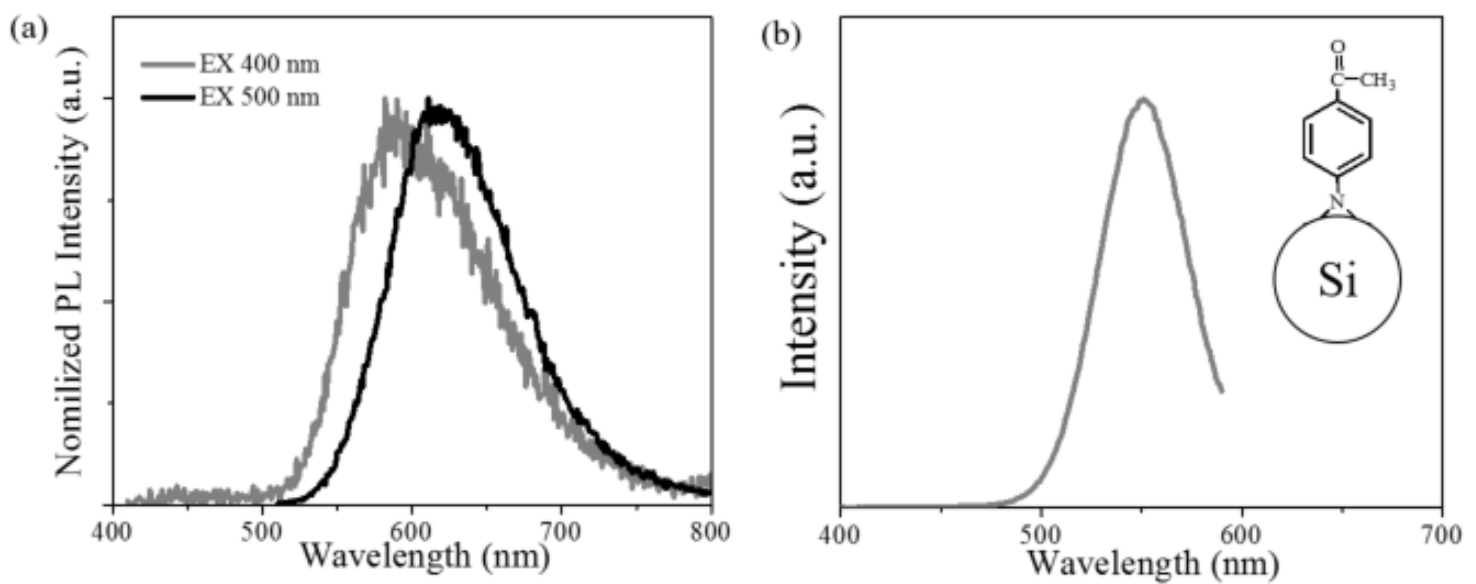

Figure S9. PL and PLE spectra of Aon-Si NPs.

(a)

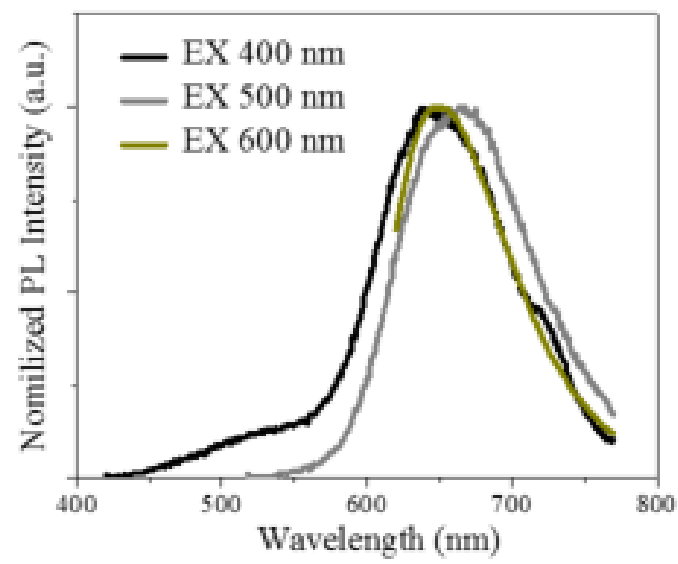

(b)

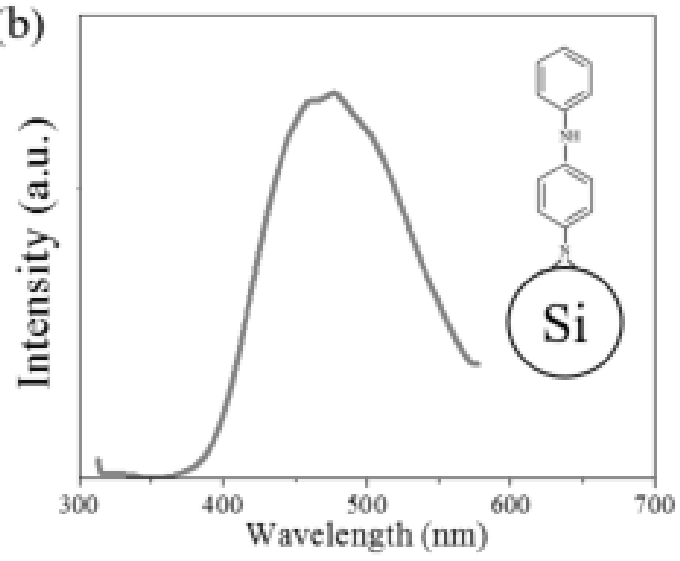

Figure S10. (a) PL and PLE spectra of the Azo-Si NPs.

(a)

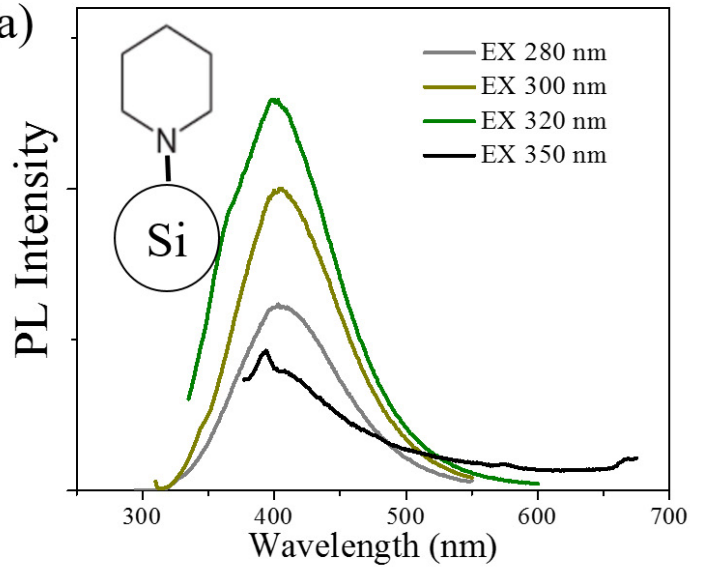

(b)

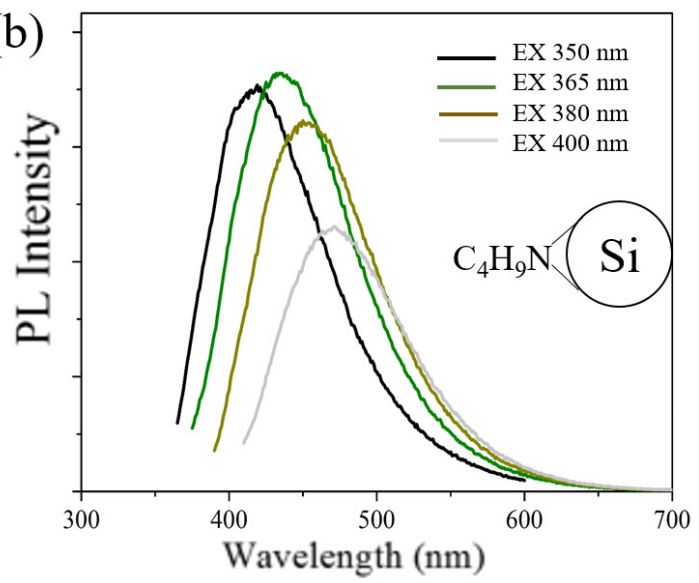

Figure S11. PL spectra of piperidine and 1-butylamine modified Si NPs. 
(a)

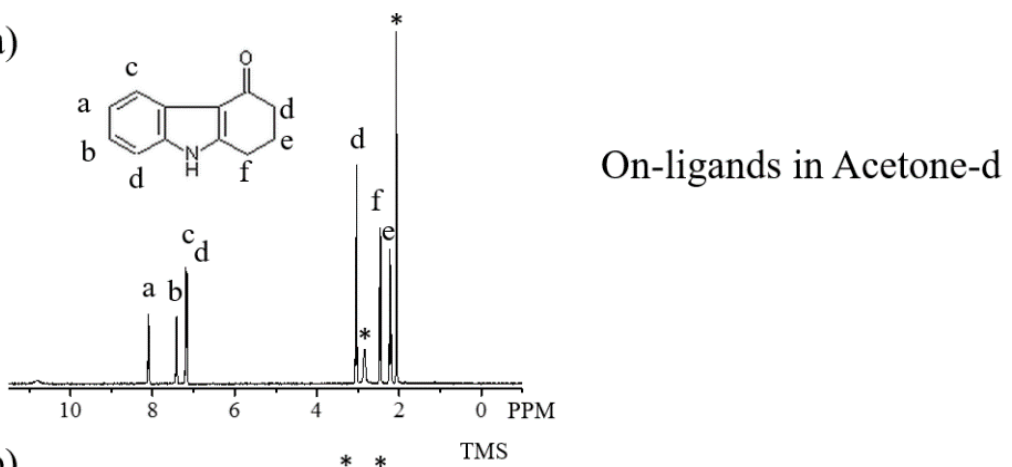

(b)

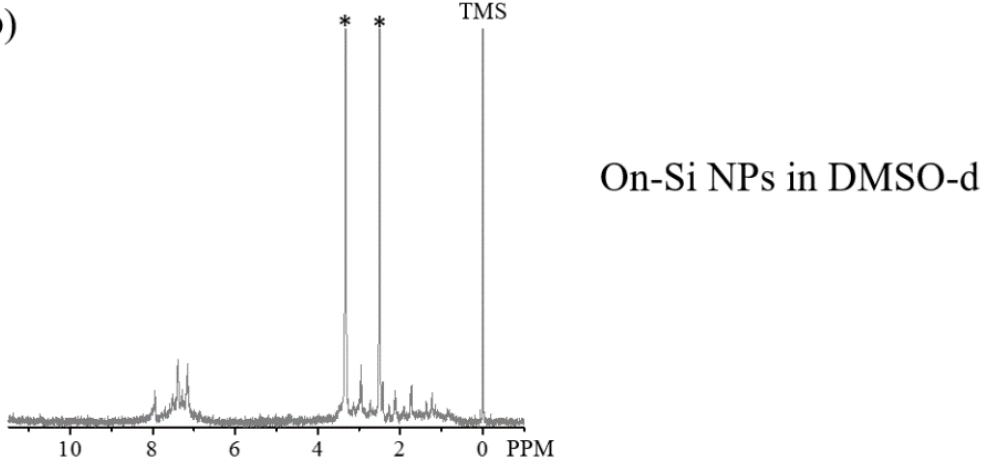

Figure S12. NMR spectra of the neat On ligands and On-Si NPs. NMR solvent peaks are denoted by asterisks $(*)$.

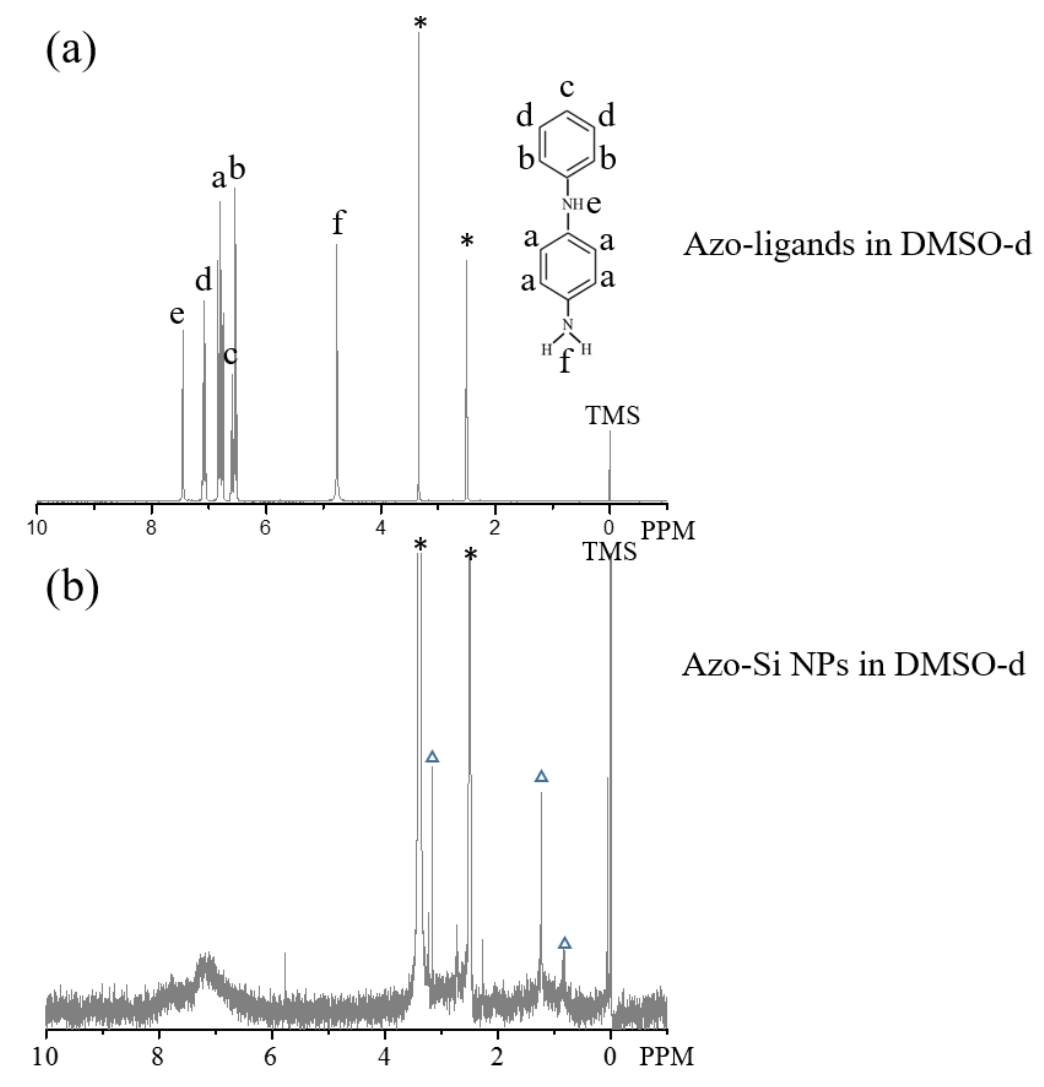

Figure S13. NMR spectra of the neat Azo ligands and Azo-Si NPs. NMR solvents denoted by asterisks $(*)$. Solvent impurities (glyme, hexane) denoted by $(\Delta)$. 


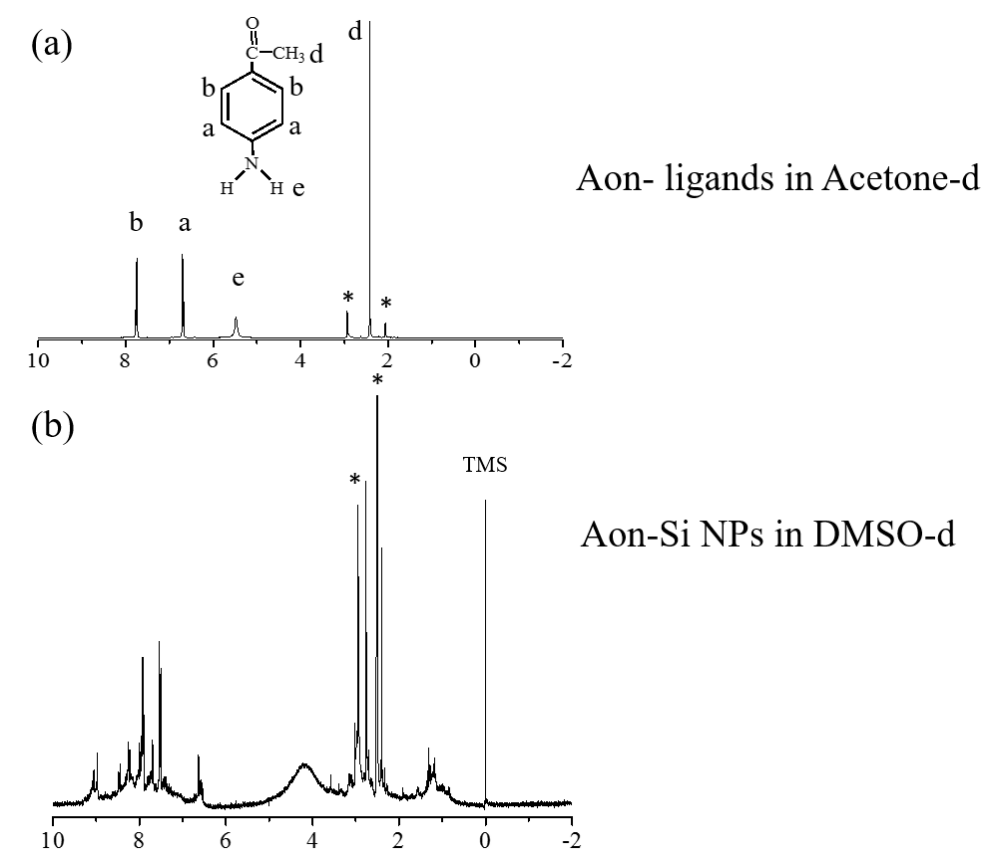

Figure S14. NMR spectra of the neat Aon ligands and Aon-Si NPs. NMR solvents denoted by asterisks $(*)$.

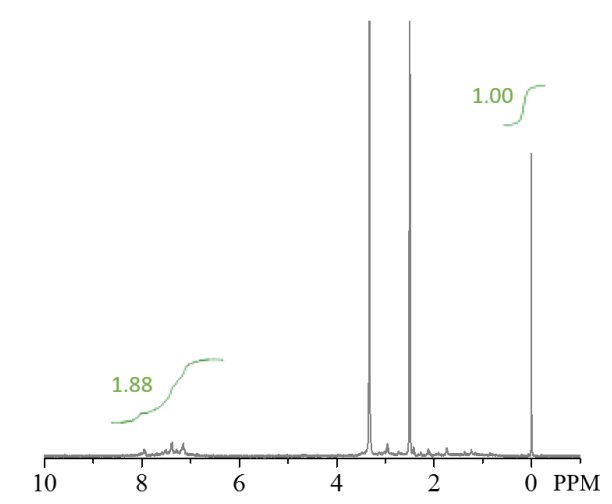

Figure S15. ${ }^{1} \mathrm{H}$ NMR spectrum of $7 \mathrm{mg}$ On-Si NPs in $1 \mathrm{~mL}$ DMSO-d6 containing $0.05 \%$ $(\mathrm{v} / \mathrm{v})$ TMS. Integration of the aromatic protons $(4 \mathrm{H})$ from On ligands relative to TMS protons $(12 \mathrm{H})$ is displayed.

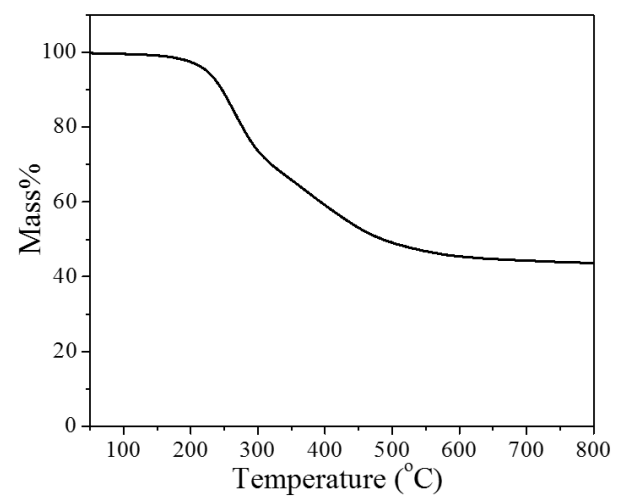

Figure S16. TGA plot of On-Si NPs (heating rate: $15^{\circ} \mathrm{C} / \mathrm{min}$, under $\mathrm{N}_{2}$ ). 


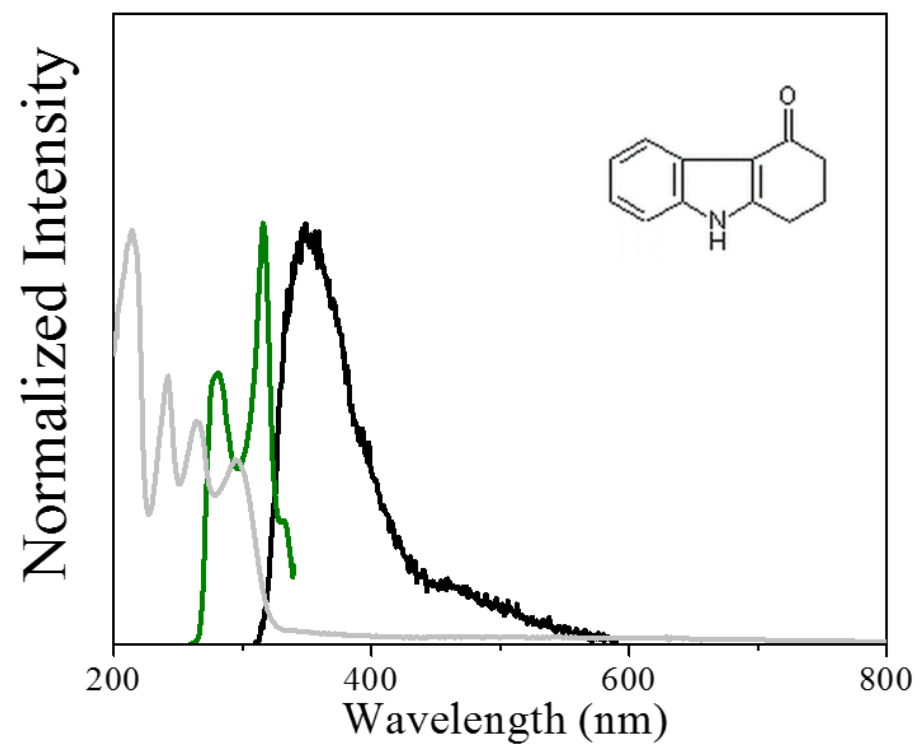

Figure S17. PL (black profile), PLE (green) and Abs (grey) spectra of the neat On ligand.

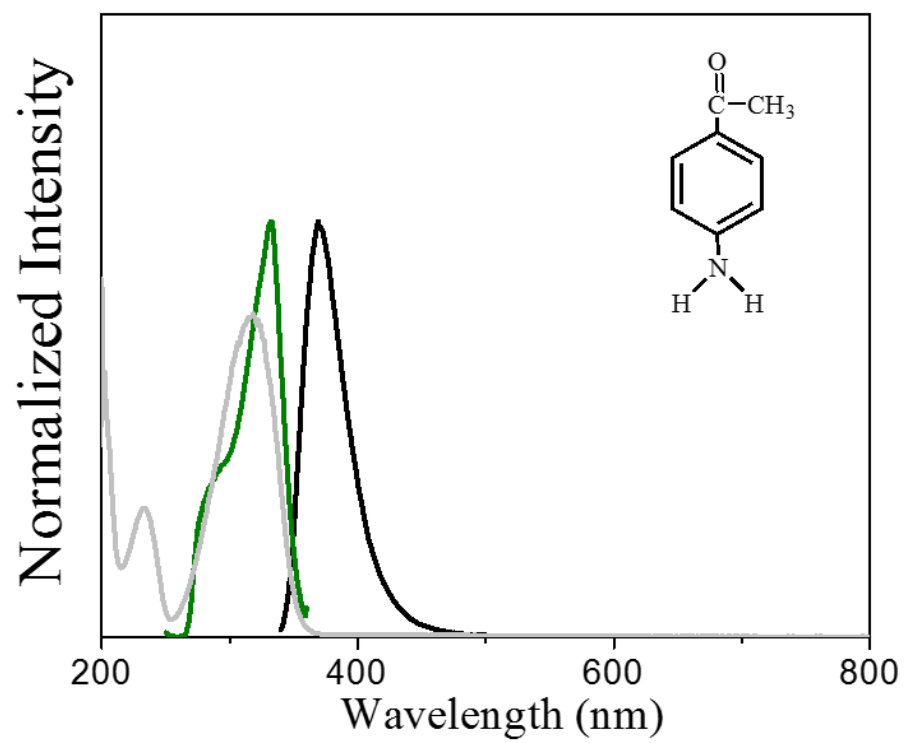

Figure S18. PL (black profile), PLE (green) and Abs (grey) spectra of the neat Aon ligand. 


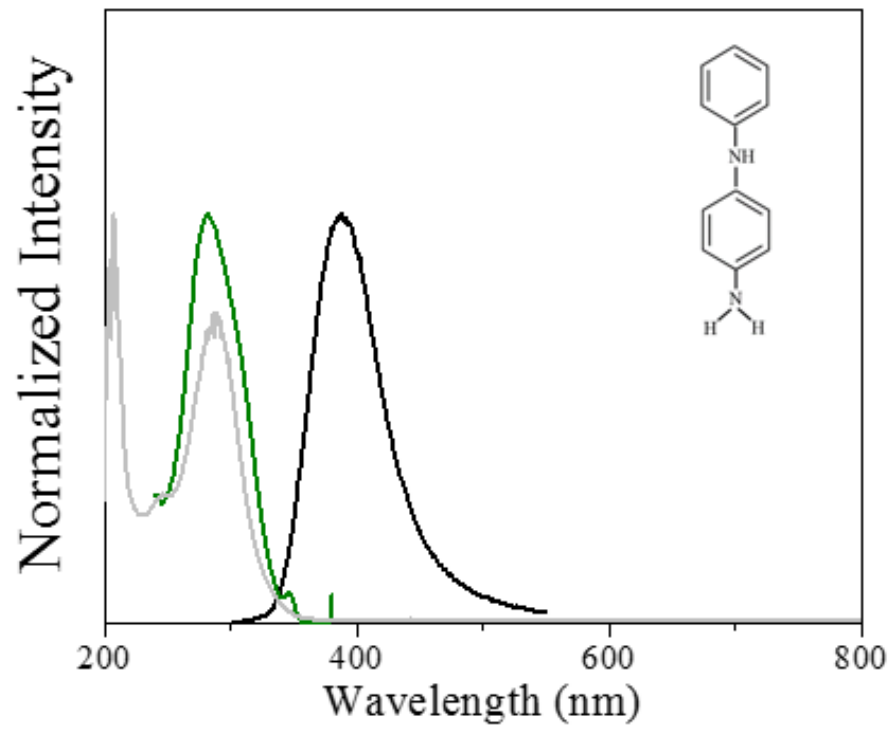

Figure S19. PL (black profile), PLE (green) and Abs (grey) spectra of the neat Azo ligand.

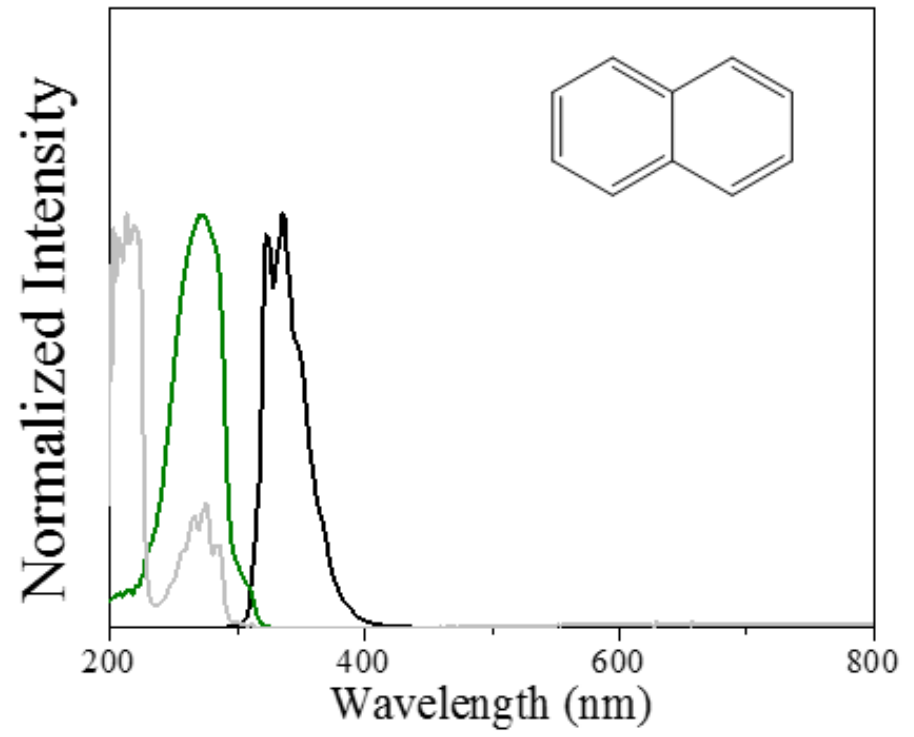

Figure S20. PL (black profile), PLE (green) and Abs (grey) spectra of the neat naphthalnene. 


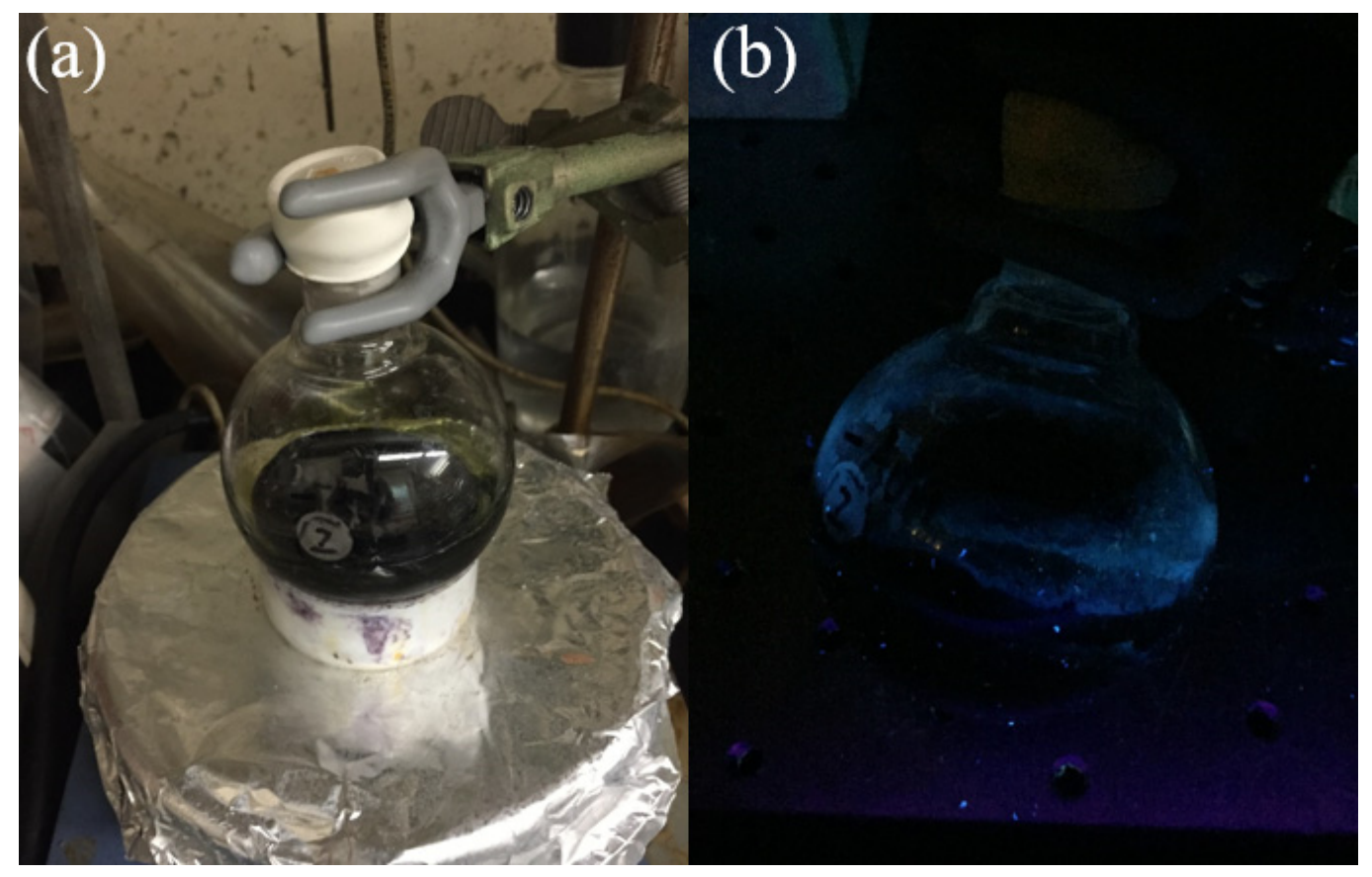

Figure S21. Photographs of the product obtained by a blank reaction using mixed glyme, $\mathrm{Na}$ naphthalide and the capping agent alone taken under room light (a) and UV (b)

A)

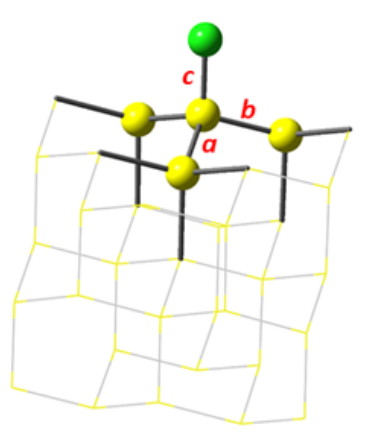

B)

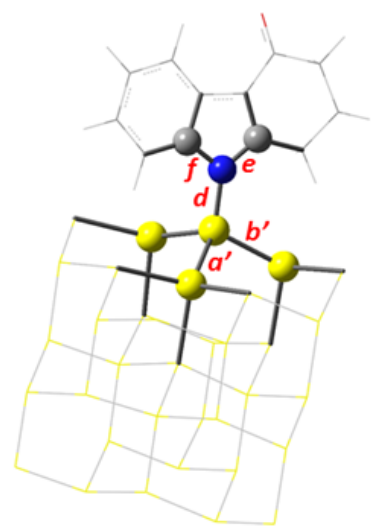

C)

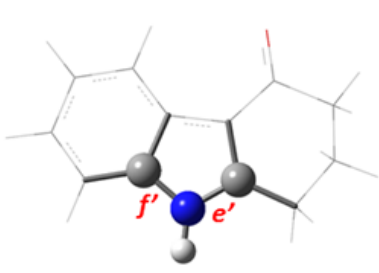

Figure S22. Optimized molecular structure of (A) $\mathrm{Si}_{35} \mathrm{H}_{36}$, (B) $\mathrm{Si}_{35} \mathrm{H}_{35} \mathrm{~L}$ and (C) L, where $\mathrm{L}=$ 1,2,3,4-tetrahydrocarbazol-4-one. The values of inter-atomic distances are: $\mathrm{a}=2.37, \mathrm{~b}=2.37$, $\mathrm{c}=2.07, \mathrm{a}^{\prime}=2.50, \mathrm{~b}^{\prime}=2.41, \mathrm{~d}=1.80, \mathrm{e}=1.41, \mathrm{f}=1.42$, and $\mathrm{e}^{\prime}=1.36, \mathrm{f}^{\prime}=1.39 \AA$. Color code: yellow. $\mathrm{Si}$, green, $\mathrm{Cl}$; gray, $\mathrm{C}$; red, $\mathrm{O}$; light gray, $\mathrm{H}$. Other $\mathrm{Cl}$ atoms are not shown for clarity. 